\title{
Optimal Power Control Over Multiple Time-Scale Fading Channels With Service Outage Constraints
}

\author{
Subhrakanti Dey, Member, IEEE, and Jamie Evans, Member, IEEE
}

\begin{abstract}
This paper considers the power-control problem for a fading channel in an information-theoretic framework. We derive power-control schemes to optimize ergodic capacity, outage capacity, and capacity with a service outage constraint. The novelty in the paper lies in the use of a two-time-scale fading process and its implications for the channel-state information available at the transmitter.
\end{abstract}

Index Terms-Ergodic capacity, fading channels, outage capacity, power control, service outage.

\section{INTRODUCTION}

D ETERMINING the information-theoretic capacity of fading wireless channels has been an important area of research over the past decade. Interest in this area has been spurred on by the tremendous growth in mobile and wireless communications networks, from cellular systems to wireless local area networks. Importantly, many recent information-theoretic results are impacting the design of next-generation wireless networks, which will include techniques such as adaptive modulation and coding and channel-based scheduling to exploit multiuser diversity. While numerous problems arise in multiuser environments such as multiple-access channels and broadcast channels, there are still many interesting questions to be answered about the capacity of single-user fading channels. This paper addresses one such question.

There are various notions of capacity for single-user fading channels, the main ones being ergodic capacity [1], delay-limited capacity [2], and capacity versus outage probability [3], [4]. Ergodic capacity is a capacity that can be achieved by averaging over all states of an ergodic fading channel, and thus, is suitable for non-real-time traffic applications. Delay-limited capacity and capacity versus outage concepts are useful for constant-rate real-time traffic applications. An excellent survey of various information-theoretic notions for fading channels is given in [5].

Another important issue in studying the capacity of fading channels is the amount of knowledge about the channel state at

Paper approved by M. Chiani, the Editor for Transmission Systems of the IEEE Communications Society. Manuscript received November 18, 2003; revised September 15, 2004. This work was supported by the Australian Research Council (ARC). The Centre for Ultra-Broadband Information Networks (CUBIN) is an affiliated program of the National ICT Australia. This paper was presented in part at the International Symposium on Modeling and Optimization in Mobile, Ad Hoc, and Wireless Networks, Cambridge, U.K., March 2004.

The authors are with the ARC Special Research Centre for Ultra-Broadband Information Networks (CUBIN), Department of Electrical and Electronic Engineering, University of Melbourne, Victoria 3010, Australia (e-mail: s.dey@ee.unimelb.edu.au; jse@ee.unimelb.edu.au).

Digital Object Identifier 10.1109/TCOMM.2005.844935 the transmitter [we will always assume that the receiver has full channel-state information (CSI)]. If the transmitter has no CSI, then all it can do is transmit with constant power. However, if it had access to CSI, then the transmit power could be controlled as a function of the channel to maximize the capacity. The paper [1] (see also [6]) looked at the problem of maximizing ergodic capacity subject to an average power constraint, and showed that the optimal power-control law was waterfilling on the inverse of the channel gain (more power is allocated when the channel is good than when the channel is bad). Another problem that suggests itself is to design power-allocation policies that minimize outage probability on a given fading channel. This problem (among others) was addressed in [4], where it was shown that the best power-allocation scheme was to use no transmit power if the channel is below a threshold, and to use channel inversion above the threshold (more power is allocated when the channel is bad than when the channel is good).

The power-allocation policies resulting from maximizing ergodic capacity and from minimizing the probability of outage are very different, and represent two ends of the spectrum. A natural question to ask is whether there is an optimization problem that bridges the gap between these two fundamental problems and their resulting optimal power-control schemes. This is exactly the question tackled in [7]. In [7], an optimal power- and rate-allocation problem is considered, where long-term average capacity is optimized with respect to a deterministic power-allocation policy, subject to a constraint on outage along with the standard average power constraint. This additional outage constraint was motivated by the idea that in an integrated network, non-real-time applications will benefit from maximizing the ergodic capacity, and at the same time, real-time applications (such as voice and video) will benefit from a quality of service (QoS) guarantee on the maximum outage probability. The optimal power allocation for this problem was shown to be a mixture of channel inversion and waterfilling allocation. Extensions of this problem to parallel fading channels with random power-allocation policies (to include discrete fading distributions) have been considered in [8].

In our paper, we generalize these results to a class of fading channels that have a two-time-scale nature. The slow variation in the fading channel is due to distance-based attenuation and shadow fading, and the resulting slow-fading channel gain is known at both transmitter and receiver. The fast-fading gain (resulting from local mobility and multipath fading) is assumed to be known at the receiver but unknown at the transmitter, however, the transmitter does have access to the statistics of the fast fading. In this paper, we restrict our discussion to the case of fast Rayleigh fading where the fast-fading gain is exponentially 
distributed. Under long codeword and sufficiently long transmission-time assumptions (as in [7]), we define a block-ergodic capacity (BEC) for each code block by averaging over the fast fading. Then we consider an optimum power- and rate-allocation problem, where the long-term average of this BEC (averaged over the slow-fading component) is maximized subject to an average power constraint and an outage constraint on the BEC. The transmitted power is now a function of the slowfading gain and the statistics of the fast-fading component. We also provide results for the related ergodic capacity and outage capacity problems.

We show that the optimum power-allocation policy is a combination of a "soft waterfilling" policy and channel inversion. We show that (similar to [7]) the resulting long-term average capacity achieves a nice compromise between the corresponding ergodic capacity and outage capacity. We also provide a suboptimal solution to the problem which has a simple power-allocation policy, but achieves near-optimal performance, verified through simulation studies. While the proof techniques are similar to those of [7], there are a number of unique contributions made in this paper.

- Derivation of a "soft waterfilling" power-allocation policy that maximizes ergodic capacity for a two-time-scale fading channel with fast Rayleigh fading.

- Derivation of an optimum power-allocation policy for the problem of maximizing ergodic capacity for a twotime-scale fading channel with fast Rayleigh fading, subject to an average power constraint and an outage constraint.

- Derivation of a simple suboptimal policy that results in near-optimal performance (demonstrated through simulation studies).

- Proofs of a number of inequalities involving the "exponential integral" not reported anywhere else, that are used to derive some intermediate results of this paper.

Before proceeding, we will discuss some related literature dealing with two-time-scale fading, and also the connection of our work to the problem of designing transmission-control schemes based on partial CSI.

The separation of the fading channel into the product of two processes evolving independently at different time scales is standard in wireless channel modeling [9]. The design of power-allocation policies based on knowledge of the slow fading has been considered by many authors (see, for example, [10]-[14] and references therein). Most of these papers deal with cellular systems and consider problems related to meeting average signal-to-interference ratio (SIR) or bit-error rate (BER) constraints, or constraints on the outage probability (which, in this case, means the probability that the SIR drops below a threshold, or equivalently, the BER rises above a threshold).

The problem of two-time-scale fading where the transmitter has access to the slow-fading component only is an example of partial CSI at the transmitter. Many authors have considered problems where the transmitter has partial CSI (see, for example, [15]-[20]). This partial CSI could take the form of a noisy estimate of the channel, a quantized version of the channel, or channel mean and covariance information in a multiple-antenna setting, to name a few. In our case, the slow-fading gain is the short-term average power of the fast-fading process, and the transmitter thus has knowledge of the local statistics of the fast fading, rather than the fast-fading gain itself. In our case, the local statistics are themselves modeled as a random process, and we look at capacities averaged over this variation.

The rest of the paper is organized as follows. Section II describes the two-time-scale fading-channel model that we use in this paper. Various capacity notions for this fading-channel model are also introduced. We also provide the problem statements for the various different optimal power-allocation tasks we are interested in. Section III presents solutions to these optimization problems. Section IV presents some simulation studies to compare the various capacity results achieved by these powerallocation algorithms. Section V presents some concluding remarks. In order to maintain readability, proofs of our results are relegated to the Appendices or excluded by alluding to similar analyses in [7].

\section{Channel Model AND VARIOUS CAPACITY Notions}

In this paper, we work with a two-time-scale version of the block flat-fading additive white Gaussian noise (BF-AWGN) channel [3] where the channel gain $h$ between the transmitter and the receiver is expressed as $h=g f, h>0$. The gain $g(>$ 0 ) represents the slow variation of the wireless radio channel, and remains constant over a block of $N$ symbols (one block is spanned by one codeword and $N$ is assumed to be large), but varies from block to block. The gain $f(>0)$ represents the fast variation of the channel and varies at a much faster rate than the rate of codeword blocks, which implies that the block length is much bigger than the coherence time of the channel. We assume that $g$ has a continuous distribution function $Q(g)$, is ergodic over the time scale of the application concerned, and is independent of the fast-fading process $f$. The fast-fading process $f$ is assumed to be exponentially distributed (assuming Rayleigh fading) with $E(f)=1$. It is reasonable to assume that both transmitters and receivers have perfect knowledge of the slow-fading gain $g$, but the transmitter does not have the knowledge of $f$. The transmitter power is assumed to be a deterministic function of $g$, and is denoted by $p(g)$. Thus, it makes sense to define the following conditional maximum achievable rate $R_{b}(g p(g))$ over each block, termed the BEC

$$
R_{b}(g p(g))=E\left[\frac{1}{2} \cdot \log _{2}\left(1+\frac{g f p(g)}{\sigma^{2}}\right) \mid g\right]
$$

where $\sigma^{2}$ is the variance of the background white noise, and the conditional expectation $E$ denotes the expectation over the distribution of the fast-fading process $f$, given $g$. In the following, "log" will denote natural logarithm.

Lemma 2.1: The BEC is given as

$$
R_{b}(g p(g))=b_{0} e^{x} E_{1}(x) \quad x=\frac{\sigma^{2}}{g p(g)}
$$

where $b_{0}=1 /(2 \log 2)$ and

$$
E_{1}(x)=\int_{x}^{\infty} \frac{e^{-t}}{t} d t
$$


Remark 1: Notice that the BEC $R_{b}(g p(g))$ is an increasing function of $p(g)$, as one would intuitively expect.

The various capacity notions that are used in the paper are presented below.

- Ergodic capacity: The ergodic capacity is defined as the expected value of the BEC, the expectation being taken over the probability density function (pdf) $q(g)$ of the slow-fading gain $g$. In other words, ergodic capacity

$$
R_{e}=E\left[R_{b}(g p(g))\right]=b_{0} E\left[e^{x} E_{1}(x)\right] \quad x=\frac{\sigma^{2}}{g p(g)} .
$$

$\bullet$

Outage capacity: The outage capacity is defined as the maximum achievable BEC over any slow-fading block, denoted by $C_{\epsilon}^{b}\left(P_{\mathrm{av}}\right)$, where

$$
C_{\epsilon}^{b}\left(P_{\mathrm{av}}\right)=\max _{p(g)} r
$$

subject to the constraints $P\left(R_{b}(g p(g))<r\right) \leq \epsilon$, $E[p(g)] \leq P_{\mathrm{av}}$, and $p(g) \geq 0$.

- The outage probability for a given BEC $r_{0}$ is defined as

$$
O(p(g))=P\left(R_{b}(g p(g))<r_{0}\right) .
$$

In what follows, we shall solve the following optimization problems.

P1) Maximize $R_{e}$ with respect to $p(g)$ subject to $E[p(g)] \leq$ $P_{\mathrm{av}}, p(g) \geq 0$.

P2) $\quad$ Maximize $R_{e}$ with respect to $p(g)$ subject to $E[p(g)] \leq$ $P_{\mathrm{av}}, p(g) \geq 0$, and $P\left(R_{b}(g p(g))<r_{0}\right) \leq \epsilon$ for a given basic BEC $r_{0}$.

P3) Maximize $r$ with respect to $p(g)$ subject to $P\left(R_{b}(g p(g))<r\right) \leq \epsilon, E[p(g)] \leq P_{\mathrm{av}}, p(g) \geq 0$, or alternatively,

P3a) Minimize the outage probability $P\left(R_{b}(g p(g))<r_{0}\right)$ with respect to $p(g)$ given a BEC $r_{0}$, subject to $E[p(g)] \leq P_{\mathrm{av}}, p(g) \geq 0$.

\section{Optimum Rate AND POWER Allocation}

In this section, we present the optimum power- and rate-allocation solutions to the problems stated above. We first provide the reader with a "roadmap" of the following Sections III-A, III-B, and III-D that are individually devoted to solving Problems P1, P2, and P3, respectively. A suboptimal solution to Problem $\mathbf{P 2}$ is also provided in Section III-C. It should be noted that the solution to $\mathbf{P 1}$ is needed in order to solve Problem P2, therefore, the first subsection presents the solution to Problem P1, followed by Section III-B that presents the solution to Problem P2. Problem P3 (or P3a) can be solved independently of $\mathbf{P 1}$ or P2. However, in the single-channel case, the solution to $\mathbf{P 3}$ is rather straightforward, and is given briefly in the last subsection. Recall that P1 finds the optimal power-control solution to maximize ergodic capacity subject to an average power constraint, $\mathbf{P 2}$ finds the optimal power-control solution to maximize ergodic capacity subject to an average power and a service outage constraint, and $\mathbf{P 3}$ finds the optimal power-control solution to maximize outage capacity (given a specified level of service outage) subject to an average power constraint. Section III-C presents a suboptimal solution to Problem P2 that is easier to implement than the optimal solution in Sec- tion III-B. Later, all these different capacity results achieved by the power-control solutions presented in the various subsections are compared via extensive numerical and simulation studies. Below, each individual subsection carries an introductory paragraph on an overview of the rationale behind the solution methodologies used and the relevance of the technical results derived in that subsection.

\section{A. Problem P1}

In this section, we provide a solution to the optimal power-control problem P1 using Lagrange-multiplier-based constraint-optimization techniques. Recall that in the absence of fast fading, it is obvious that the solution to $\mathbf{P 1}$ is the well-known waterfilling solution [21]. In order to apply the usual Lagrangian-based constrained-optimization theory, we first show (in Lemma 3.1) that the objective function in Problem $\mathbf{P 1}$ is a concave function of $p(g)$ (note: it is easy to see that the constraint functions are linear in $p(g)$ ). It is then verified that the optimal power-control solution to $\mathbf{P 1}$ is obtained as a unique fixed-point solution (as a deterministic function of the slow-fading gain $g$, when $g$ is above a certain threshold) to an implicit equation, and can be computed numerically using an iterative algorithm with guaranteed convergence. It is also seen that when the slow-fading gain falls below this threshold (which is determined by the average power available), the optimal strategy is to turn off transmission. Some key properties of this power-control policy are then presented, as they will be useful in subsequent mathematical analyses. Noticing the similarity between this solution and the well-known waterfilling power-control policy for the slow-fading channel case, we call the optimal power-control solution to Problem P1 soft waterfilling. We conclude this subsection with a few comments on the differences between these two policies.

As discussed above, we need the following result in order to proceed (see Appendix III-A for a proof).

Lemma 3.1: The BEC $R_{b}(g(p(g))$ is a concave function of $p(g)$.

Remark 2: One can, of course, deduce the above result simply by appealing to the fact that $\log \left(1+\left(g f p(g) / \sigma^{2}\right)\right)$ is a concave function of $p(g)$, and the expectation of this function taken over the distribution of $f$ (under the Rayleigh fading assumption) will retain the concavity, a result that follows by the Dominated Convergence Theorem.

Lemma 3.1 indicates clearly that $R_{e}$ is also a concave function of $p(g)$, again by appealing to the Dominated Convergence Theorem, under some mild conditions satisfied by the distribution of the slow fading gain $g$.

Recalling that the constraints of $\mathbf{P 1}$ are linear in $p(g)$, one can now apply the usual Lagrangian-based constrained-optimization method to form the Lagrangian

$$
L\left(p(g), g, \lambda_{0}\right)=\left[R_{b}(g p(g))-\lambda_{0} \frac{b_{0}}{\sigma^{2}} p(g)\right] q(g)
$$

where $q(g)$ is the pdf of $g$ and $\lambda_{0}>0$.

Lemma 3.2: The fixed-point equation

$$
p^{*}\left(g, \lambda_{0}\right)=\frac{\sigma^{2}}{\lambda_{0}}\left(1-y e^{y} E_{1}(y)\right), \quad y=\frac{\sigma^{2}}{g p^{*}\left(g, \lambda_{0}\right)}
$$




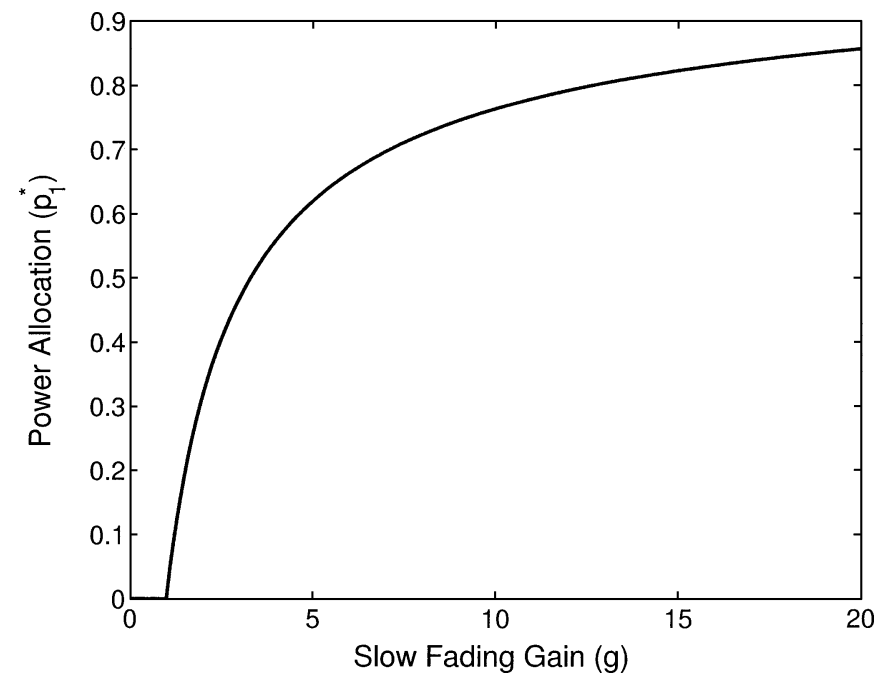

Fig. 1. Plot of soft waterfilling allocation versus the slow-fading gain.

has a unique positive fixed-point solution for every $g>\lambda_{0}$.

Remark 3: The unique fixed-point solution to (6) (for a given value of $g$ ) can be computed numerically via an iterative algorithm that is convergent. The details of this algorithm should be obvious from the proof of Lemma 3.2 (see Appendix II), and are excluded.

Definition 3.1: Suppose for any given $g>\lambda_{0}, p_{1}^{*}\left(g, \lambda_{0}\right)>0$ is the unique fixed point of $(6)$. Define $p_{1}^{*}\left(g, \lambda_{0}\right)=0$ for $0 \leq$ $g \leq \lambda_{0}$.

It can be easily verified that $p_{1}^{*}\left(g, \lambda_{0}\right)$ satisfies the Kuhn-Tucker conditions for optimality (see [22, p. 74] for details). Hence, the solution to the optimization problem P1 is given by $p_{1}^{*}\left(g, \lambda_{0}\right)$, as defined above. The multiplier $\lambda_{0}$ is evaluated by solving $E\left[p_{1}^{*}\left(g, \lambda_{0}\right)\right]=P_{\mathrm{av}}$. Some key properties of $p_{1}^{*}\left(g, \lambda_{0}\right)$ are summarized below.

Lemma 3.3: The optimal power-allocation solution to Problem P1 $\left[p_{1}^{*}\left(g, \lambda_{0}\right)\right]$ satisfies the following properties: 1) $p_{1}^{*}\left(g, \lambda_{0}\right)$ is a strictly increasing function of $g$ for $g>\lambda_{0}$; and 2) $p_{1}^{*}\left(g, \lambda_{0}\right) \rightarrow 0$ as $g \rightarrow \lambda_{0}$ from above, and $p_{1}^{*}\left(g, \lambda_{0}\right) \rightarrow\left(\sigma^{2} / \lambda_{0}\right)$ as $g \rightarrow \infty$.

Now we discuss some properties of this optimal solution $p_{1}^{*}\left(g, \lambda_{0}\right)$, in comparison with the waterfilling solution one would obtain in the absence of fast fading. Recall that the waterfilling solution is given by $p_{w}^{*}\left(g, \lambda_{w}\right)=\sigma^{2}\left(\left(1 / \lambda_{w}\right)-(1 / g)\right)^{+}$, where $\lambda_{w}$ is the Lagrange multiplier that solves $E\left[p_{w}^{*}\left(g, \lambda_{w}\right)\right]=$ $P_{\mathrm{av}}$ and $(x)^{+}=\max (0, x)$. Both $p_{1}^{*}\left(g, \lambda_{0}\right)$ and $p_{w}^{*}\left(g, \lambda_{w}\right)$ are zero for values of $g$ below a threshold. In addition, they are both increasing functions of $g$ above this threshold, and approach a limit as $g \rightarrow \infty$. We have observed that $p_{1}^{*}\left(g, \lambda_{0}\right)$ tends to increase to the limit more gradually than the waterfilling solution, so, for want of a better name, we call the solution to Problem P1 "soft" waterfilling.

Fig. 1 shows a plot of $p_{1}^{*}(g, \lambda)$ versus the slow fading gain $g$ for $\lambda=1$ with $\sigma^{2}=1$.

\section{B. Problem P2}

In this section, we derive the optimum power-allocation policy for Problem P2. This problem, as correctly pointed out in [7], is difficult because of the probabilistic constraint involving the outage probability. One way to circumvent this problem is to consider a probabilistic power-allocation policy, as in [4]. In this paper, however, we focus on a deterministic power-allocation policy. As in [7], we proceed by first deriving a solution to a similar problem (Problem P2a), but with a deterministic constraint on the BEC $R_{b}(g p(g))$. This constraint requires that the $\mathrm{BEC}$ is greater than the minimum required rate $r_{0}$ over an arbitrary set of the slow-fading gain $g$. This problem can be solved using standard Lagrangian-multiplier-based techniques for convex optimization, as the BEC is a concave function of $p(g)$. The solution to Problem P2a not only provides us with a feasibility condition for Problem P2, but also provides us with important insight into how to obtain an optimal solution to $\mathbf{P 2}$. It is seen that the optimal power-allocation policy for $\mathbf{P 2}$ is analogous to the solution to P2a, except that this solution guarantees that $R_{b}(g p(g)) \geq r_{0}$ over a particular set of the slow-fading gain determined by the maximum service outage probability $\epsilon$. Theorem 1 summarizes the solution to P2a, while Theorem 2 provides an intermediate result. These two theorems (see Appendices for proofs) together lead to the main result of this paper, namely, the optimal solution to Problem P2, following a similar analysis in [7]. This result is stated in Theorem 3, which shows that the optimal power-allocation policy for $\mathbf{P 2}$ is a combination of channel inversion and the optimal power-allocation solution to Problem P1, namely, the soft waterfilling policy. The rest of the subsection provides the required mathematical analysis. Proofs are either relegated to the Appendices or excluded by alluding to a similar analysis in [7].

In order to proceed, we need the definition of a service set and an outage set, as in [7].

Definition 3.2: Given a basic rate $r_{0}$ and a power policy $p(g)$, the service set is defined as

$$
\mathcal{H}_{s}(p(g))=\left\{g \mid R_{b}(g p(g)) \geq r_{0}\right\} .
$$

Correspondingly, the outage set is defined as

$$
\mathcal{H}_{o}(p(g))=\left\{g \mid R_{b}(g p(g))<r_{0}\right\} .
$$

It will be seen that our solution to Problem P2 will result in a power-allocation policy having a particular form of a service set (mirroring a similar result in [7]). In order to derive this result, we first solve the following problem, where it is required that the service set contains an arbitrary set $\mathcal{H}_{r}$.

Problem P2a: Find $R^{*}\left(\mathcal{H}_{r}\right)=\max _{p(g)} R_{e}$ subject to

$$
\begin{aligned}
E(p(g)) & \leq P_{\mathrm{av}} \\
p(g) & \geq 0 \\
R_{b}(g p(g)) & \geq r_{0} \quad \forall g \in \mathcal{H}_{r} .
\end{aligned}
$$

It is obvious (by noting that $e^{x} E_{1}(x)$ is a monotonically decreasing function of $x$ ) that a feasible solution $p(g)$ to Problem P2a has to satisfy

$$
p(g) \geq \frac{\sigma^{2}}{g x_{0}} \quad g \in \mathcal{H}_{r}
$$


where $e^{x_{0}} E_{1}\left(x_{0}\right)=r_{0} / b_{0}$. This implies that for Problem P2a to be feasible, a necessary condition is $P_{\mathrm{av}} \geq P_{\min }\left(r_{0}, \mathcal{H}_{r}\right)$ where

$$
P_{\min }\left(r_{0}, \mathcal{H}_{r}\right)=\int_{\mathcal{H}_{r}} \frac{\sigma^{2}}{g x_{0}} q(g) d g .
$$

Let us denote an optimal solution to Problem P2a by $p^{*}\left(g, \mathcal{H}_{r}\right)$. Following the usual Lagrange-multiplier-based convex-optimization theory, one obtains the following solution to Problem P2a (see Appendix IV for a proof).

Theorem 1: Suppose $P_{\mathrm{av}}=P_{\min }\left(r_{0}, \mathcal{H}_{r}\right)$. Then the optimal power-allocation solution to Problem P2a is given by

$$
p^{*}\left(g, \mathcal{H}_{r}\right)= \begin{cases}\frac{\sigma^{2}}{g x_{0}}, & g \in \mathcal{H}_{r} \\ 0, & \text { otherwise. }\end{cases}
$$

If $P_{\mathrm{av}}>P_{\min }\left(r_{0}, \mathcal{H}_{r}\right)$, then the optimal power-allocation solution to Problem P2a is given by

$$
p^{*}\left(g, \mathcal{H}_{r}\right)= \begin{cases}\frac{\sigma^{2}}{g x_{0}}, & g \in \mathcal{H}_{r} \cap\{g \leq \bar{g}\} \\ p_{1}^{*}\left(g, \lambda_{1}\right), & \text { otherwise }\end{cases}
$$

where $\bar{g}=\lambda_{1} /\left(x_{0}\left(1-x_{0} r_{0} / b_{0}\right)\right)$, and $\lambda_{1}$ is the solution to $E\left[p^{*}\left(g, \mathcal{H}_{r}\right)\right]=P_{\mathrm{av}}$.

The interpretation of the result above is that the optimal power allocation is a combination of channel inversion over the slow-fading component and the optimal power allocation for obtaining maximum average block-ergodic rate given by the solution to Problem P1, namely, the soft waterfilling solution. Within the service set, the allocated power is no less than that achieved by inverting the slow-fading component to maintain the basic block-ergodic rate $r_{0}$. For slow-fading gains larger than a threshold $(\bar{g})$, the allocated power is larger, given by the soft waterfilling solution to achieve the maximum average block-ergodic rate.

As in [7], we now define the set of good channels and bad channels according to the value of the slow-varying channel gain $g$. Defining $Q\left(g_{\epsilon}\right)=\epsilon$, the set of good channels is defined as $\mathcal{H}_{\epsilon}=\left\{g: g \geq g_{\epsilon}\right\}$, and the set of bad channels is defined as $\overline{\mathcal{H}}_{\epsilon}=\left\{g: g<g_{\epsilon}\right\}$. We will show that the solution to Problem P2a with $\mathcal{H}_{r}=\mathcal{H}_{\epsilon}$, i.e, $p^{*}\left(g, \mathcal{H}_{\epsilon}\right)$, is an optimum solution to Problem P2.

Define the partial ordering of two sets, as in [7], that is, $\mathcal{H}_{1} \prec$ $\mathcal{H}_{2}$ if $g_{1}<g_{2}, \forall g_{1} \in \mathcal{H}_{1}$ and $g_{2} \in \mathcal{H}_{2}$. Then one can prove the following theorem.

Theorem 2: Problem $\mathbf{P 2}$ has an optimum solution $p^{*}(g)=p^{*}\left(g, \mathcal{H}_{\epsilon}\right)$ with the outage set $\mathcal{H}_{o}\left(p^{*}(g)\right)$ and service set $\mathcal{H}_{s}\left(p^{*}(g)\right)$, such that $\mathcal{H}_{o}\left(p^{*}(g)\right) \prec \mathcal{H}_{s}\left(p^{*}(g)\right)$.

Proof: The proof is based on a complex two-stage construction process, and is relegated to Appendix V.

This theorem basically mirrors the corresponding result in [7, Th. 2] in that it implies that the block-ergodic rate falls below the basic rate $r_{0}$ when the slowly varying channel gain falls below a particular threshold.

Now using the fact that $P\left(\mathcal{H}_{s}\left(p^{*}(g)\right)\right) \geq 1-\epsilon$, one can easily show that Problem $\mathbf{P} 2$ has an optimum solution $p^{*}(g)=$ $p^{*}\left(g, \mathcal{H}_{\epsilon}\right)$, such that $\mathcal{H}_{\epsilon} \subseteq \mathcal{H}_{s}\left(p^{*}(g)\right)$. A similar analysis to [7] now leads to the main result of this paper.
Theorem 3: Problem $\mathbf{P 2}$ has a solution if and only if $\left(P_{\mathrm{av}}, r_{0}, \epsilon\right)$ satisfies $P_{\mathrm{av}} \geq P_{\min }\left(r_{0}, \mathcal{H}_{\epsilon}\right)$. When $P_{\mathrm{av}}=P_{\min }$ $\left(r_{0}, \mathcal{H}_{\epsilon}\right)$, the optimum power allocation is given by

$$
p^{*}(g)= \begin{cases}\frac{\sigma^{2}}{g x_{0}}, & g \in \mathcal{H}_{\epsilon} \\ 0, & \text { otherwise. }\end{cases}
$$

When $P_{\text {av }}>P_{\min }\left(r_{0}, \mathcal{H}_{\epsilon}\right)$, an optimum power allocation is given by

$$
p^{*}(g)= \begin{cases}\frac{\sigma^{2}}{g x_{0}}, & g_{\epsilon} \leq g \leq \max \left(g_{\epsilon}, g^{*}\right) \\ p_{1}^{*}\left(g, \lambda^{*}\right), & \text { otherwise }\end{cases}
$$

where $\bar{g}^{*}=\lambda^{*} / x_{0}\left(1-x_{0} r_{0} / b_{0}\right)$, and $\lambda^{*}$ is the solution to $E\left[p^{*}\left(g, \mathcal{H}_{\epsilon}\right)\right]=P_{\mathrm{av}}$.

Note that computing $p^{*}(g)$ involves computing $p_{1}^{*}\left(g, \lambda^{*}\right)$, which has to be computed numerically (for a given value of $g$ ) as the unique fixed-point solution to an implicit equation, as given by (6). In order to avoid this computational complexity, one may be motivated to look at a suboptimal solution to Problem P2. This is the topic of the following subsection.

\section{A Suboptimal Solution to Problem P2}

In this section, we present a simple suboptimal solution to Problem $\mathbf{P 2}$ by noticing that

$$
E\left[E\left[\log \left(1+\frac{g f p(g)}{\sigma^{2}}\right) \mid g\right]\right] \leq E\left[\log \left(1+\frac{g p(g)}{\sigma^{2}}\right)\right]
$$

by Jensen's inequality, remembering that $E[f]=1$. Now one can pose the following optimization task.

Problem P2b: Maximize $E\left[\log \left(1+\left(g p(g) / \sigma^{2}\right)\right)\right]$ with respect to $p(g)$, subject to $E[p(g)] \leq P_{\mathrm{av}}$ and $p(g) \geq 0$ and $P\left(R_{b}(g p(g))<r_{0}\right) \leq \epsilon$.

Notice that this problem can be treated in a similar way to that of [7] by observing that the constraint $P\left(R_{b}(g p(g))<r_{0}\right) \leq \epsilon$ can be rewritten as $P(p(g))<\left(\sigma^{2} / g x_{0}\right) \leq \epsilon$.

An optimal solution to Problem P2b can now be obtained in exactly the same fashion as in [7] (hence, we do not provide the analysis), and we call this solution $p_{\text {sub }}(g)$. The next theorem states this result.

Theorem 4: A suboptimal solution to Problem P2, given as an optimum solution to $\mathbf{P} \mathbf{2 b}$, is given by [if $P_{\mathrm{av}}=P_{\min }$ $\left.\left(r_{0}, \mathcal{H}_{\epsilon}\right)\right]$

$$
p_{\text {sub }}(g)= \begin{cases}\frac{\sigma^{2}\left(2^{2 r_{1}}-1\right)}{g}, & g \in \mathcal{H}_{\epsilon} \\ 0, & \text { otherwise. }\end{cases}
$$

Otherwise, if $P_{\mathrm{av}}>P_{\min }\left(r_{0}, \mathcal{H}_{\epsilon}\right)$

$$
p_{\mathrm{sub}}(g)= \begin{cases}\frac{\sigma^{2}\left(2^{2 r_{1}}-1\right)}{g}, & g_{\epsilon} \leq g \leq \max \left(g_{\epsilon}, g_{\mathrm{sub}} * 2^{2 r_{1}}\right) \\ \sigma^{2}\left(\frac{1}{g_{\mathrm{sub}}}-\frac{1}{g}\right)^{+}, & \text {otherwise }\end{cases}
$$

where $r_{1}=b_{0} \log \left(1+\left(1 / x_{0}\right)\right.$ and $g_{\text {sub }}$ is the solution to $E\left[p_{\mathrm{sub}}(g)\right]=P_{\mathrm{av}}$.

Later, we will compare the capacity results achieved by the optimal and suboptimal solutions to $\mathbf{P 2}$ via simulation studies.

\section{Problem $\mathbf{P 3}$}

In this section, we provide the optimal solution to Problems P3a and P3. Note that the proof is intuitively straightforward in the case of a single communication channel, with the 
two-time-scale fading properties satisfying the assumptions in Section II, and the power-allocation policy being deterministic. Because of this, we only provide a brief sketch of a proof of Theorem 5. In addition, by noting that the service outage probability can also be written as $P\left(g p(g)<\left(\sigma^{2} / x_{0}\right)\right)$ (where $\left.e^{x_{0}} E_{1}\left(x_{0}\right)=r_{0}\right)$, it can be easily seen that the solution to Problem P3 can be obtained as a special case of the outage-capacity maximization problem for multiple parallel channels considered in [4], where the number of channels is one. The corresponding optimal power-allocation problem for outage capacity maximization, in the case of multiple parallel communication channels with two-time-scale fading properties (where we can also allow the slow-fading gain to be a discrete random process and the power-allocation policy to be probabilistic), is complex and is beyond the scope of this paper. This problem will be treated rigorously in a subsequent paper.

Theorem 5: The outage probability is minimized by the optimal power-allocation solution to Problem P3a

$$
p_{\text {out }}(g)= \begin{cases}\frac{\sigma^{2}}{g x_{0}}, & g>g_{\text {out }} \\ 0, & \text { otherwise }\end{cases}
$$

where $\int_{g_{\text {out }}}^{\infty}\left(\sigma^{2} / g x_{0}\right) q(g) d g=P_{\text {av }}$. Similarly, the outage capacity is given by $\bar{r}$, such that $\bar{r}=b_{0} \exp (\bar{x}) E_{1}(\bar{x})$ and $\bar{x}$ is given by $\int_{q_{\epsilon}}^{\infty}\left(\sigma^{2} / g \bar{x}\right) q(g) d g=P_{\mathrm{av}}$.

Proof: It is easy to show that the BEC within the service set should be exactly $r_{0}$, since if it is greater than $r_{0}$ for some subset of the service set, the service set could be expanded by redistributing power [since the BEC is an increasing function of $p(g)$ ], such that the average power constraint is satisfied, thus reducing outage probability. Similarly, the optimal powerallocation solution $p_{\text {out }}(g)$ must also satisfy the average power constraint with equality, otherwise additional power could be invested in enlarging the service set. The particular form of the solution $p_{\text {out }}(g)$, as given above by Theorem 5, thus follows easily.

\section{Simulation Results}

In this section, we present some simulation studies conducted with a single-user channel. The slow-fading gain $g$ is distributed with a lognormal distribution (standard for shadow fading), such that $\log g$ is distributed with mean 0 and variance $\sigma_{g}=10^{0.3}$. The fast-fading gain $f$ is exponentially distributed with mean 1 (Rayleigh fading). The minimum basic rate is taken to be $r_{0}=$ $1 \mathrm{~b} / \mathrm{s} / \mathrm{Hz}$. The maximum outage probability is constrained to be $\epsilon=0.05 .\left(P_{\mathrm{av}} / \sigma^{2}\right)$ is varied between 11 and $14 \mathrm{~dB}$.

Fig. 2 shows how the various capacity measures increase with increasing signal-to-noise ratio (SNR). The SNR is computed as $10 \log _{10}\left(P_{\mathrm{av}} / \sigma^{2}\right)$. As it can be seen, capacity with outage achieves a compromise between ergodic capacity and outage capacity, the gap between ergodic capacity and capacity with outage decreasing with increasing average transmission power. What is interesting is to observe that the suboptimal power-allocation solution $p_{\mathrm{sub}}(g)$ results in nearly optimal performance. Thus, one can use the easily computable solution $p_{\text {sub }}(g)$ (as opposed to $p^{*}(g)$ ) in order to achieve good capacity performance with the same outage constraint.

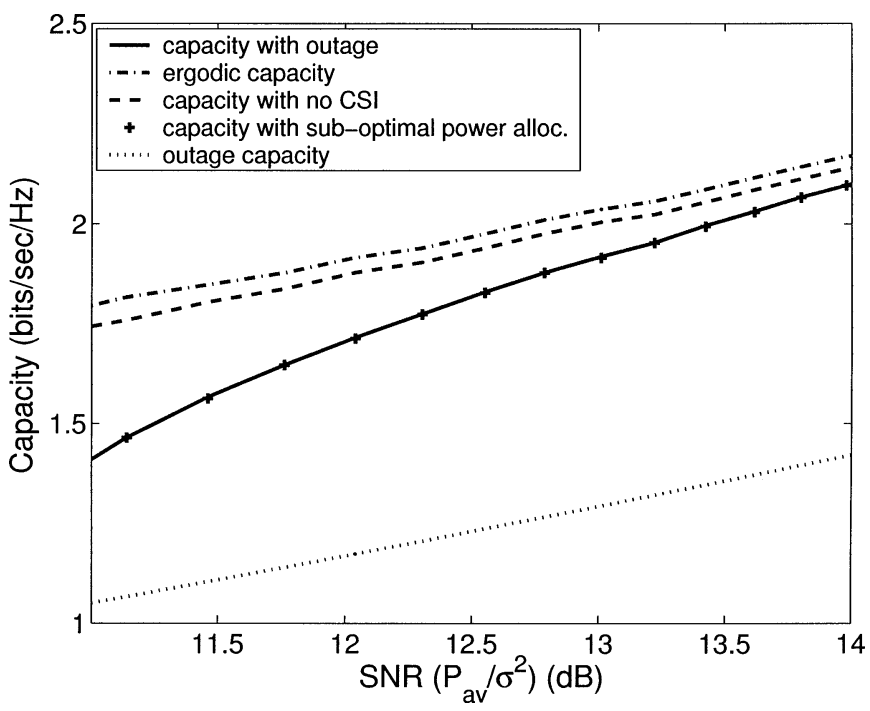

Fig. 2. Various capacity plots against $P_{\text {av }} / \sigma^{2}$.

\section{CONCLUSION}

In this paper, we have studied the power-control problem for wireless channels from an information-theoretic perspective. Integral to the developments was the use of a two-time-scale fading model which incorporated slow fading due to distance and shadowing effects, and fast fading due to multipath propagation. We assumed that the transmitter had knowledge of the slowfading gain and the statistic of the fast fading, but did not know the instantaneous value of the fast-fading component. Power-control schemes were thus designed to exploit knowledge of the slow fading. We considered various notions of capacity, including ergodic capacity, outage capacity, and capacity subject to a service outage constraint, developing optimal powerallocation strategies in each case.

Extensions of this work are currently being considered for parallel channels, as well as to the case of multiaccess channels with applications to opportunistic scheduling.

\section{APPENDIX I}

\section{PROOF OF LEMMA 3.1}

In this proof, to keep notations simple, we use $R_{b}$ instead of $R_{b}(g p(g))$. It is easy to work out that [with $x=\left(\sigma^{2} / g p(g)\right)$ ]

$$
\begin{aligned}
& \frac{d^{2} R_{b}}{d p^{2}(g)}=-\frac{b_{0}}{p^{2}(g)}\left[\left(1-x e^{x} E_{1}(x)\right)\right. \\
&\left.+p(g)\left\{(x+1) e^{x} E_{1}(x)-1\right\}\right] .
\end{aligned}
$$

Using the fact that $p(g) \geq 0$, and the two standard inequalities $e^{x} E_{1}(x) \leq(1 / x), e^{x} E_{1}(x)>(1 /(x+1))$ for $x>0$, it follows that $\left(d^{2} R_{b} / d p^{2}(g)\right)<0$. Thus $R_{b}(g(p(g))$ is a concave function of $p(g)$.

\section{APPENDIX II \\ PROOF OF LEMMA 3.2}

Notice that (6) can be written as

$$
\frac{\lambda_{0}}{g}=y\left[1-y e^{y} E_{1}(y)\right] .
$$


Define $q_{1}(y)=y\left[1-y e^{y} E_{1}(y)\right]$. First, one can easily check that for $y=0, q_{1}(y)=0$. Notice that

$$
\frac{d q}{d y}=1+y-y e^{y} E_{1}(y)(y+2) .
$$

Using the continued fraction expansion of $e^{y} E_{1}(y)$ (see [23]), one can write for $y>0$

$$
y e^{y} E_{1}(y)=\frac{1}{1+\frac{1}{y+\frac{1}{1+\cdots}}}<\frac{1}{1+\frac{1}{y+1}}=\frac{y+1}{y+2}
$$

which implies that $(d q / d y)>0$, for $y>0$. Therefore, $q_{1}(y)=$ $y\left[1-y e^{y} E_{1}(y)\right]$ is a strictly monotonically increasing function for $y>0$. Also, it is straightforward to show that $q_{1}(y)<1$ for $y>0$ and $q_{1}(y) \rightarrow 1$ as $y \rightarrow \infty$. Therefore, for every given $g>\lambda_{0}$, (6) has a unique fixed-point solution $p^{*}\left(g, \lambda_{0}\right)>0$.

\section{APPENDIX III}

\section{PROOF OF LEMMA 3.3}

1)We need the following proposition involving the exponential integral function $E_{1}(x)$.

Proposition 1: For $x>0$

$$
\frac{(x+1) e^{x} E_{1}(x)-1}{\frac{1}{x}-e^{x} E_{1}(x)}
$$

is an increasing function of $x$. As $x \rightarrow \infty$

$$
\frac{(x+1) e^{x} E_{1}(x)-1}{\frac{1}{x}-e^{x} E_{1}(x)} \rightarrow 1 .
$$

It also follows that

$$
\frac{(x+1) e^{x} E_{1}(x)-1}{\frac{1}{x}-e^{x} E_{1}(x)}<1
$$

for $0<x<\infty$.

Proof: In this proof, we assume $x>0$. Let us denote

$$
V(x)=\frac{(x+1) e^{x} E_{1}(x)-1}{\frac{1}{x}-e^{x} E_{1}(x)} .
$$

By evaluating $d V(x) / d x$ one gets that a necessary condition for $d V(x) / d x>0$ is

$$
\left[e^{x} E_{1}(x)-\frac{3 x+1}{2 x^{2}}\right]^{2}<\frac{x^{2}+6 x+1}{4 x^{4}}
$$

which implies [noting that $e^{x} E_{1}(x)<(1 / x)$ ]

$$
\frac{3 x+1}{2 x^{2}}>e^{x} E_{1}(x)>\frac{3 x+1-\sqrt{x^{2}+6 x+1}}{2 x^{2}} .
$$

The inequality of the left-hand side (LHS) is trivial to prove, since $e^{x} E_{1}(x)<(1 / x)$. The right-hand side (RHS) inequality is rather tricky to prove. One proof based on a continued fraction expansion of $e^{x} E_{1}(x)$ [24] is as follows:

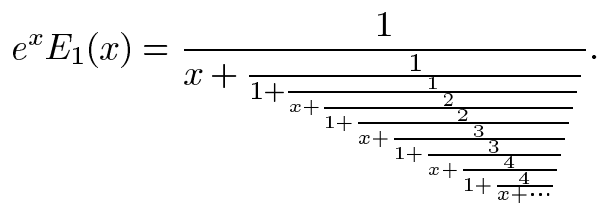

It is then straightforward to show (working upwards from the lowest level shown) that

$$
\begin{aligned}
e^{x} E_{1}(x) & >\frac{x^{3}+15 x^{2}+58 x+50}{x^{4}+16 x^{3}+72 x^{2}+96 x+24} \\
& >\frac{(3 x+1)-\sqrt{x^{2}+6 x+1}}{2 x^{2}}
\end{aligned}
$$

where the first inequality follows from the continued fraction expansion, and the second inequality follows after some tedious algebraic manipulation. This implies that

$$
\frac{(x+1) e^{x} E_{1}(x)-1}{\frac{1}{x}-e^{x} E_{1}(x)}
$$

is an increasing function of $x$ for $x>0$.

Notice also trivially that

$$
\frac{(x+1) e^{x} E_{1}(x)-1}{\frac{1}{x}-e^{x} E_{1}(x)}>0
$$

for $x>0$. Using the asymptotic expansion for $e^{x} E_{1}(x)$ [23], one can show that

$$
\frac{(x+1) e^{x} E_{1}(x)-1}{\frac{1}{x}-e^{x} E_{1}(x)} \rightarrow 1
$$

as $x \rightarrow \infty$. This also implies that

$$
\frac{(x+1) e^{x} E_{1}(x)-1}{\frac{1}{x}-e^{x} E_{1}(x)}<1
$$

for $x>0$.

From Lemma 3.2, $p_{1}^{*}\left(g, \lambda_{0}\right)$ satisfies the following equation for $g>\lambda_{0}$ :

$$
p_{1}^{*}\left(g, \lambda_{0}\right)=\frac{\sigma^{2}}{\lambda_{0}}\left(1-x e^{x} E_{1}(x)\right) \quad x=\frac{\sigma^{2}}{g p_{1}^{*}\left(g, \lambda_{0}\right)} .
$$

This implies that for $g>\lambda_{0}$

$$
\begin{aligned}
\frac{d p_{1}^{*}\left(g, \lambda_{0}\right)}{d g}= & \frac{\sigma^{2}}{\lambda_{0}}\left[1-(x+1) e^{x} E_{1}(x)\right] \frac{d x}{d g} \\
= & \frac{x^{2}}{\lambda_{0}}\left[(x+1) e^{x} E_{1}(x)-1\right] \\
& \times\left(p_{1}^{*}\left(g, \lambda_{0}\right)+g \frac{d p_{1}^{*}\left(g, \lambda_{0}\right)}{d g}\right) .
\end{aligned}
$$

Rearranging, one can write

$$
\begin{aligned}
\frac{d p_{1}^{*}\left(g, \lambda_{0}\right)}{d g}\left[1-\frac{x^{2}}{\lambda_{0}}\{(\right. & \left.\left.x+1) e^{x} E_{1}(x)-1\right\} g\right] \\
& =\frac{x^{2}}{\lambda_{0}} p_{1}^{*}\left(g, \lambda_{0}\right)\left\{(x+1) e^{x} E_{1}(x)-1\right\} .
\end{aligned}
$$

Since $e^{x} E_{1}(x)>(1 /(x+1))$, it is easy to see that the RHS of the above equation is positive. We now show that

$$
\left[1-\frac{x^{2}}{\lambda_{0}}\left\{(x+1) e^{x} E_{1}(x)-1\right\} g\right]>0 .
$$

By rearranging (16), one can easily show that the LHS of the previous inequality is identical to

$$
1-\frac{(x+1) e^{x} E_{1}(x)-1}{\frac{1}{x}-e^{x} E_{1}(x)} .
$$


It can be shown that

$$
\frac{(x+1) e^{x} E_{1}(x)-1}{\frac{1}{x}-e^{x} E_{1}(x)}<1
$$

for $x>0$ (see Proposition 1). Thus, $\left(d p_{1}^{*}\left(g, \lambda_{0}\right) / d g\right)>0$ for $g>\lambda_{0}$ and 1) is proved.

2) As $g$ increases, $x$ decreases [from part 1)], hence $x e^{x} E_{1}(x) \rightarrow 0$ as $g \rightarrow \infty$. Therefore, $p_{1}^{*}\left(g, \lambda_{0}\right) \rightarrow\left(\sigma^{2} / \lambda_{0}\right)$.

Similarly, as $g \rightarrow \lambda_{0}$ from above, $p_{1}^{*}\left(g, \lambda_{0}\right)$ decreases strictly monotonically. As $p_{1}^{*}\left(g, \lambda_{0}\right) \geq 0, p_{1}^{*}\left(g, \lambda_{0}\right) \rightarrow 0$ as $g \rightarrow \lambda_{0}$.

\section{APPENDIX IV \\ PROOF OF THEOREM 1}

When $P_{\mathrm{av}}=P_{\min }\left(r_{0}, \mathcal{H}_{r}\right)$, solution (8) is obvious. When $P_{\mathrm{av}}>P_{\min }\left(r_{0}, \mathcal{H}_{r}\right)$, the problem can be translated to the following problem.

Maximize $E\left[R_{b}(g p(g))\right]$ with respect to $p(g)$ subject to $E[p(g)]=P_{\mathrm{av}}, p(g) \geq 0$ and $p(g) \geq\left(\sigma^{2} / g x_{0}\right), g \in \mathcal{H}_{r}$.

Notice that from Lemma 3.1, $R_{b}(g p(g))$ is a concave function of $p(g)$. Therefore, Problem P2a has a concave objective function and linear or concave constraints. Then $p^{*}\left(g, \mathcal{H}_{r}\right)$ is the optimal solution iff it satisfies the Kuhn-Tucker conditions [25].

Using a Lagrange multiplier $b_{0} \lambda_{1}>0$, we define the Lagrangian

$$
L\left(p(g), g, \lambda_{1}\right)=\left[R_{b}(g p(g))-\lambda_{1} b_{0} p(g)\right] q(g) .
$$

Define $\overline{\mathcal{H}}_{g}$ to be the union of the sets $\left\{g \in \mathcal{H}_{r} \mid p^{*}\left(g, \mathcal{H}_{r}\right)>\right.$ $\left.\left(\sigma^{2} / g x_{0}\right)\right\}$ and $\left\{g \notin \mathcal{H}_{r} \mid p^{*}\left(g, \mathcal{H}_{r}\right)>0\right\}$. It is then straightforward to show that when $g \in \overline{\mathcal{H}}_{g}$

$$
\left.\frac{d L\left(p(g), g, \lambda_{1}\right)}{d p(g)}\right|_{p(g)=p^{*}\left(g, \mathcal{H}_{r}\right)}=0
$$

and

$$
\left.\frac{d L\left(p(g), g, \lambda_{1}\right)}{d p(g)}\right|_{p(g)=p^{*}\left(g, \mathcal{H}_{r}\right)} \leq 0
$$

when $g \notin \overline{\mathcal{H}}_{g}$. Since $p^{*}\left(g, \mathcal{H}_{r}\right)$ is unique and positive $\forall g>\lambda_{1}$ (Lemma 3.2), and $p^{*}\left(g, \mathcal{H}_{r}\right)=0$ for $g \leq \lambda_{0}$, the Kuhn-Tucker conditions are satisfied. Therefore, $p^{*}\left(g, \mathcal{H}_{r}\right)$ is the optimum solution to Problem P2a.

\section{APPENDIX V \\ PROOF OF THEOREM 2}

The proof of this theorem is based on a two-stage construction process provided in [7]. To avoid repetition, we summarize most of the proof. However, there are certain intermediate results needed, which are not straightforward to prove. More details are provided for these results.

Suppose there is an arbitrary feasible power policy for Problem $\mathbf{P 2}$ given by $\hat{p}(g)$ with service set $\hat{\mathcal{H}}_{s}$ and long-term average rate $\hat{R}$, with $E[\hat{p}(g)] \leq P_{\text {av }}$ and $P\left(\hat{\mathcal{H}}_{s}\right) \geq 1-\epsilon$. Then, another policy $\tilde{p}(g)$ can be constructed by letting $\mathcal{H}_{r}=\hat{\mathcal{H}}_{s}$ in Problem P2a, such that [for $P_{\text {av }}>P_{\min }\left(r_{0}, \hat{\mathcal{H}}_{s}\right)$ ]

$$
\tilde{p}(g)= \begin{cases}\frac{\sigma^{2}}{g x_{0}}, & g \in \hat{\mathcal{H}}_{s} \cap\{g \leq \tilde{g}\} \\ p_{1}^{*}(g, \tilde{\lambda}), & \text { otherwise }\end{cases}
$$

where $\tilde{g}=\tilde{\lambda} /\left(x_{0}\left(1-x_{0} r_{0} / b_{0}\right)\right)$ and $\tilde{\lambda}$ is the solution to $E[\tilde{p}(g)]=P_{\mathrm{av}}$. For $P_{\mathrm{av}}=P_{\min }\left(r_{0}, \hat{\mathcal{H}}_{s}\right)$

$$
\tilde{p}(g)= \begin{cases}\frac{\sigma^{2}}{g x_{0}}, & g \in \mathcal{H}_{r} \\ 0, & \text { otherwise. }\end{cases}
$$

$\tilde{p}(g)$ is obviously feasible and achieves a higher long-term average rate than $\hat{R}$, since $\tilde{p}(g)$ achieves the highest average rate among all policies whose service sets contain $\hat{\mathcal{H}}_{s}$.

Now define the following power policies over the entire space of the slow-fading gain $g \in(0, \infty)$ :

$$
\begin{aligned}
\tilde{p}_{\text {swf }}(g) & =p_{1}^{*}(g, \tilde{\lambda}) \quad 0<g<\infty \\
\tilde{p}_{\text {res }}(g) & =\left[\frac{\sigma^{2}}{g x_{0}}-\tilde{p}_{\text {swf }}(g)\right]^{+} \quad 0<g<\infty .
\end{aligned}
$$

Also define the set (over which $\left.R_{b}(g \tilde{p}(g)) \geq r_{0}\right) \tilde{\mathcal{H}}_{\text {swf }}=\{g$ : $g \geq \tilde{g}\}$ and $\tilde{\mathcal{H}}_{\text {inv }}=\hat{\mathcal{H}}_{s} \backslash \tilde{\mathcal{H}}_{\text {swf }}$. Notice that $\tilde{p}(g)$ can also be written as

$$
\tilde{p}(g)=\tilde{p}_{\text {swf }}(g)+1_{g \in \tilde{\mathcal{H}}_{\text {inv }}} \tilde{p}_{\text {res }}(g)
$$

where $1_{x}$ is the indicator function taking value 1 when $x$ is true, and 0 otherwise. Now construct another power-allocation policy

$$
p^{\prime}(g)=\tilde{p}_{\mathrm{swf}}(g)+1_{g \in \mathcal{H}_{\text {inv }}^{\prime}} \tilde{p}_{\text {res }}(g)
$$

where $\mathcal{H}_{\mathrm{inv}}^{\prime}=\left\{g_{b}^{\prime} \leq g<\tilde{g}\right\}$ and

$$
\int_{\mathcal{H}_{\text {inv }}^{\prime}} \tilde{p}_{\text {res }}(g) q(g) d g=\operatorname{int}_{\tilde{\mathcal{H}}_{\text {inv }}} \tilde{p}_{\text {res }}(g) q(g) d g .
$$

Denote the average rates achieved by $\tilde{p}(g)$ and $p^{\prime}(g)$ as $\tilde{R}$ and $R^{\prime}$, respectively. Then, we have the following lemma.

Lemma 5.1: The power-allocation policy $p^{\prime}(g)$ has the following properties:

1) $E\left[p^{\prime}(g)\right]=E[\tilde{p}(g)]=P_{\mathrm{av}}$;

2) $\mathcal{H}_{o}\left(p^{\prime}(g)\right) \prec \mathcal{H}_{s}\left(p^{\prime}(g)\right)$;

3) $R^{\prime} \geq \tilde{R}$;

4) $P\left\{\overline{\mathcal{H}}_{s}\left(p^{\prime}(g)\right)\right\} \geq P\left\{\mathcal{H}_{s}(\tilde{p}(g))\right\}$.

Proof: Proofs of 1) and 2) are trivial (see [7]) for details. Proofs of 3) and 4) require Proposition 2 and Proposition 4. The proof of Proposition 2 is algebraically rather complex and is provided in sufficient detail, whereas Proposition 4 is directly quoted from [7].

Proposition 2: The power-efficiency function given by

$$
\eta(g)=\frac{r_{0}-R_{b}\left(g \tilde{p}_{\text {swf }}(g)\right)}{\tilde{p}_{\text {res }}(g)} \quad 0<g<\tilde{g}
$$

is an increasing function of $g$. 
Proof: Using the fact that $\tilde{p}_{\text {swf }}(g)$ satisfies (6), one can (after some algebraic manipulation) rewrite $1 / \eta(g)$ as

$$
\beta(x)=c_{1} \frac{\left(x-x_{0}\right)\left(1-x e^{x} E_{1}(x)\right)}{e^{x_{0}} E_{1}\left(x_{0}\right)-e^{x} E_{1}(x)}
$$

where $c_{1}$ is a generic constant. For $g<\tilde{g}, x>x_{0}$ as at $g=$ $\tilde{g}, \tilde{p}_{\text {swf }}(g)=\sigma^{2} / g x_{0}$. Now we need to show that $\beta(x)$ is an increasing function of $x$ for $x>x_{0}$ where $x_{0}>0$. Denoting $\alpha(x)=\left(x-x_{0}\right)\left(1-x e^{x} E_{1}(x)\right)$ and $\gamma(x)=e^{x_{0}} E_{1}\left(x_{0}\right)-$ $e^{x} E_{1}(x)$, notice that at $\alpha\left(x_{0}\right)=\gamma\left(x_{0}\right)=0$. Note also that both $\alpha(x)$ and $\gamma(x)$ are positive for $x>x_{0}$. It is obvious that $\gamma(x)$ is an increasing function of $x$ for $x>x_{0}$. The same can be said about $\alpha(x)$, by observing that $(d / d x) \alpha(x)=x[(1 / x-$ $\left.\left.e^{x} E_{1}(x)\right)-\left((x+1) e^{x} E_{1}(x)-1\right)\right]+x_{0}$, which is positive from Proposition 1 and the fact $x_{0}>0$.

Now it is straightforward to derive that

$$
\begin{aligned}
& \frac{\frac{d}{d x} \alpha(x)}{\frac{d}{d x} \gamma(x)}=x\left[1-\frac{(x+1) e^{x} E_{1}(x)-1}{\frac{1}{x}-e^{x} E_{1}(x)}\right] \\
& +x_{0}\left[\frac{(x+1) e^{x} E_{1}(x)-1}{\frac{1}{x}-e^{x} E_{1}(x)}\right] .
\end{aligned}
$$

Clearly, from Proposition 1

$$
\frac{\frac{d}{d x} \alpha(x)}{\frac{d}{d x} \gamma(x)}>0 .
$$

We also know from Proposition 1 that

$$
\frac{(x+1) e^{x} E_{1}(x)-1}{\frac{1}{x}-e^{x} E_{1}(x)}
$$

is an increasing function of $x$ for $x>0$. Hence, we just need to show that

$$
x\left[1-\frac{(x+1) e^{x} E_{1}(x)-1}{\frac{1}{x}-e^{x} E_{1}(x)}\right]
$$

is an increasing function of $x$ for $x>0$, which is the following proposition.

Proposition 3: For $x>0$

$$
x\left[1-\frac{(x+1) e^{x} E_{1}(x)-1}{\frac{1}{x}-e^{x} E_{1}(x)}\right]
$$

is an increasing function of $x$.

Proof: The proof of this is similar to that of Proposition 1. Writing

$$
W(x)=x\left[1-\frac{(x+1) e^{x} E_{1}(x)-1}{\frac{1}{x}-e^{x} E_{1}(x)}\right]
$$

one can (after some algebra) show that $(d W(x) / d x)>0$ implies

$$
\left(e^{x} E_{1}(x)\right)^{2} 2(x+1)-e^{x} E_{1}(x)\left(5+\frac{4}{x}\right)+\frac{3 x+1}{x^{2}}>0 .
$$

This implies

$$
\left[e^{x} E_{1}(x)-\frac{5 x+4}{4 x(x+1)}\right]^{2}>\frac{x^{2}+8 x+8}{4 x(x+1)^{2}} .
$$

Noting again that

$$
\frac{5 x+4}{4 x(x+1)}>\frac{1}{x}>e^{x} E_{1}(x)
$$

one needs to now show that

$$
e^{x} E_{1}(x)<\frac{5 x+4-\sqrt{x^{2}+8 x+8}}{4 x(x+1)}
$$

for $x>0$. The proof of this is again based on the continued fraction expansion (15), which leads to

$$
\begin{aligned}
e^{x} E_{1}(x) & <\frac{x^{3}+11 x^{2}+26 x+6}{x\left(x^{3}+12 x^{2}+36 x+24\right)} \\
& <\frac{5 x+4-\sqrt{x^{2}+8 x+8}}{4 x(x+1)} .
\end{aligned}
$$

This completes the proof.

Therefore, we have shown that $(d / d x) \alpha(x) /(d / d x) \gamma(x)$ is a positive increasing function of $x$ for $x>x_{0}$, and the proof is complete.

Proposition 4: For two disjoint sets $\Psi_{1}, \Psi_{2}$, let $f_{1}(x)$ be an arbitrary function, such that $f_{1}\left(x^{\prime \prime}\right) \geq f_{1}\left(x^{\prime} \forall x^{\prime \prime} \in \Psi_{2}\right.$, $x^{\prime} \in \Psi_{1}$. For any nonnegative function $f_{2}(x)$ satisfying $\int_{\Psi_{2}} f_{2}(x) d x=\int_{\Psi_{1}} f_{2}(x) d x$, we have

$$
\int_{\Psi_{2}} f_{1}(x) f_{2}(x) d x \geq \int_{\Psi_{1}} f_{1}(x) f_{2}(x) d x .
$$

Now we continue our proofs of parts 3) and 4) of Lemma 5.1. Proof of 3): Define $\Psi=\tilde{\mathcal{H}}_{\text {inv }} \cap \mathcal{H}_{\text {inv }}^{\prime}$ such that $\tilde{\Psi}=$ $\tilde{\mathcal{H}}_{\text {inv }} \Psi$ and $\Psi^{\prime}=\mathcal{H}_{\text {inv }}^{\prime} \Psi$ are two disjoint sets. The average rate of $\tilde{p}(g)$ can be expressed as

$$
\begin{aligned}
\tilde{R}=\int_{0}^{\infty} R_{b}\left(g \tilde{p}_{\mathrm{swf}}(g)\right) q(g) d g & \\
& +\int_{\tilde{\mathcal{H}}_{\text {inv }}}\left(r_{0}-R_{b}\left(g \tilde{p}_{\mathrm{swf}}(g)\right)\right) q(g) d g .
\end{aligned}
$$

Using the definition of the power-efficiency function (23), one can write

$$
\tilde{R}=R_{\text {swf }}+\int_{\Psi} \eta(g) \tilde{p}_{\text {res }}(g) q(g) d g+\int_{\tilde{\Psi}} \eta(g) \tilde{p}_{\text {res }}(g) q(g) d g
$$

where $R_{\mathrm{swf}}=\int_{0}^{\infty} R_{b}\left(g \tilde{p}_{\mathrm{swf}}(g)\right) q(g) d g$. From a similar expression for $R^{\prime}$, we finally get

$$
R^{\prime}-\tilde{R}=\int_{\Psi^{\prime}} \eta(g) \tilde{p}_{\text {res }}(g) q(g) d g-\int_{\tilde{\Psi}} \eta(g) \tilde{p}_{\text {res }}(g) q(g) d g .
$$

Since $\tilde{P} s i \prec \Psi^{\prime}$ (see [7]), by Proposition 2, Proposition 4, and (22), we get $R^{\prime} \geq \tilde{R}$.

Proof of 4): By repeating the analysis in [7], it can be shown that $P\left\{\mathcal{H}_{s}\left(p^{\prime}(g)\right)\right\}-P\left\{\mathcal{H}_{s}(\tilde{p}(g))\right\}=P\left(\Psi^{\prime}\right)-P(\tilde{\Psi})$, which is equal to

$$
\int_{\Psi^{\prime}} \frac{1}{\tilde{p}_{\text {res }}(g)} \tilde{p}_{\text {res }}(g) q(g) d g-\int_{\tilde{\Psi}} \frac{1}{\tilde{p}_{\text {res }}(g)} \tilde{p}_{\text {res }}(g) q(g) d g .
$$

Note that $\tilde{p}_{\text {res }}(g)=\left(\sigma^{2} / g x_{0}\right)-\tilde{p}_{\text {swf }}(g)$ when $g \in \Psi^{\prime}$ or $g \in \tilde{\Psi}$. Since from Lemma 3.3, $\tilde{p}_{\text {swf }}(g)$ is an increasing function of $g$, it is obvious that $\tilde{p}_{\text {res }}(g)$ is a decreasing function of $g$, and hence, $\left.1 / \tilde{p}_{\text {res }}(g)\right)$ is an increasing function of $g$. Proposition 4 now implies that $P\left\{\mathcal{H}_{s}\left(p^{\prime}(g)\right)\right\} \geq P\left\{\mathcal{H}_{s}(\tilde{p}(g))\right\}$. 
Thus, starting from any arbitrary feasible power allocation $\tilde{p}(g)$, one can construct a better power-allocation policy $p^{\prime}(g)$, such that $\mathcal{H}_{o}\left(p^{\prime}(g)\right) \prec \mathcal{H}_{s}\left(p^{\prime}(g)\right)$. This proves Theorem 2 .

\section{ACKNOWLEDGMENT}

The authors would like to thank J. Luo for useful discussions.

\section{REFERENCES}

[1] A. J. Goldsmith and P. Varaiya, "Capacity of fading channels with channel side information," IEEE Trans. Inf. Theory, vol. 43, no. 11, pp. 1986-1992, Nov. 1997.

[2] S. V. Hanly and D. N. C. Tse, "Multi-access fading channels-Part II: Delay-limited capacities," IEEE Trans. Inf. Theory, vol. 44, no. 11, pp. 2816-2831, Nov. 1998.

[3] L. H. Ozarow, S. Shamai, and A. D. Wyner, "Information theoretic considerations for cellular mobile radio," IEEE Trans. Veh. Technol., vol. 43, no. 5, pp. 359-378, May 1994.

[4] G. Caire, G. Taricco, and E. Biglieri, "Optimum power control over fading channels," IEEE Trans. Inf. Theory, vol. 45, no. 7, pp. 1468-1489, Jul. 1999.

[5] E. Biglieri, J. Proakis, and S. Shamai, "Fading channels: Informationtheoretic and communications aspects," IEEE Trans. Inf. Theory, vol. 44, no. 10, pp. 2619-2692, Oct. 1998.

[6] G. Caire and S. Shamai, "On the capacity of some channels with channel state information," IEEE Trans. Inf. Theory, vol. 45, no. 9, pp. 2007-2019, Sep. 1999.

[7] J. Luo, L. Lin, R. Yates, and P. Spasojevic, "Service outage based power and rate allocation," IEEE Trans. Inf. Theory, vol. 49, no. 1, pp. 323-330, Jan. 2003.

[8] J. Luo, R. Yates, and P. Spasojevic, "Service outage based power and rate allocation for parallel fading channels,", Preprint, 2003.

[9] T. S. Rappaport, Wireless Communications: Principles and Practice. Englewood Cliffs, NJ: Prentice-Hall, 2001.

[10] S. W. Kim and A. J. Goldsmith, "Truncated power control in code-division multiple-access communications," IEEE Trans. Veh. Technol., vol. 49, no. 5, pp. 965-972, May 2000.

[11] J. Zhang, E. K. P. Chong, and I. Kontoyiannis, "Unified spatial diversity combining and power allocation for CDMA systems in multiple timescale fading channels," IEEE J. Sel. Areas Commun., vol. 19, no. 7, pp. 1276-1288, Jul. 2001.

[12] S. Vishwanath, S. A. Jafar, and A. J. Goldsmith, "Adaptive resource allocation in composite fading environments," in Proc. IEEE Global Telecommun. Conf., San Antonio, TX, Nov. 2001, pp. 1312-1316.

[13] S. Kandukuri and S. P. Boyd, "Optimal power control in interferencelimited fading channels with outage-probability specifications," IEEE Trans. Wireless Commun., vol. 1, no. 1, pp. 46-55, Jan. 2002.

[14] J. Papandriopoulos, J. S. Evans, and S. Dey, "Iterative power control and multiuser detection with outage probability constraints," in Proc. IEEE Int. Conf. Commun., Anchorage, AK, May 2003, pp. 2509-2513.

[15] P. Ligdas and N. Farvardin, "Optimizing the transmit power for slow fading channels," IEEE Trans. Inf. Theory, vol. 46, no. 2, pp. 565-576, Mar. 2000.

[16] A. Das and P. Narayan, "Capacities of time-varying multiple-access channels with side information," IEEE Trans. Inf. Theory, vol. 48, no. 1, pp. 4-25, Jan. 2002

[17] E. Visotsky and U. Madhow, "Space-time transmit precoding with imperfect feedback," IEEE Trans. Inf. Theory, vol. 47, no. 9, pp. 2632-2639, Sep. 2001.

[18] S. H. Simon and A. L. Moustakas, "Optimizing MIMO antenna systems with channel covariance feedback," IEEE J. Sel. Areas Commun., vol. 21, no. 3, pp. 406-417, Apr. 2003.

[19] P. Xia, S. Zhou, and G. B. Giannakis, "Adaptive MIMO-OFDM based on partial channel state information," IEEE Trans. Signal Process., vol. 52, no. 1, pp. 202-213, Jan. 2004.
[20] S. A. Jafar and A. J. Goldsmith, "Transmitter optimization and optimality of beamforming for multiple antenna systems," IEEE Trans. Wireless Commun., vol. 3, no. 4, pp. 1165-1175, Jul. 2004.

[21] T. Cover and J. Thomas, Elements of Information Theory. New York: Wiley, 1991.

[22] M. J. Fryer and J. V. Greenman, Optimization Theory: Applications in OR and Economics. London, U.K.: E. Arnold, 1987.

[23] M. Abramowitz and I. A. Stegun, Handbook of Mathematical Functions With Formulas, Graphs and Mathematical Tables. New York: Dover, 1974.

[24] L. Lorentzen and H. Waadeland, Continued Fractions With Applications. Amsterdam, The Netherlands: North-Holland, 1974.

[25] D. Luenberger, Optimization by Vector Space Methods. New York: Wiley, 1969

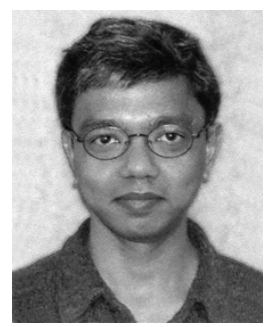

Subhrakanti Dey (S'94-M'96) was born in Calcutta, India, in 1968. He received the B.Tech. and M.Tech. degrees from the Department of Electronics and Electrical Communication Engineering, Indian Institute of Technology, Kharagpur, India, in 1991 and 1993, respectively, and the Ph.D. degree from the Department of Systems Engineering, Research School of Information Sciences and Engineering, Australian National University, Canberra, Australia, in 1996.

He has been with the Department of Electrical and Electronic Engineering, University of Melbourne, Parkville, Australia, since February 2000, first as a Senior Lecturer, and then as an Associate Professor. From September 1995 to September 1997 and September 1998 to February 2000, he was a Postdoctoral Research Fellow with the Department of Systems Engineering, Australian National University. From September 1997 to September 1998, he was a Postdoctoral Research Associate with the Institute for Systems Research, University of Maryland, College Park. His current research interests include signal processing for telecommunications, wireless communications and networks, performance analysis of communication networks, stochastic and adaptive estimation and control, and statistical and adaptive signal processing.

Dr. Dey currently serves on the Editorial Boards of the IEEE TRANSACTIONS on AutOMATIC CONTROL and Systems and Control Letters.

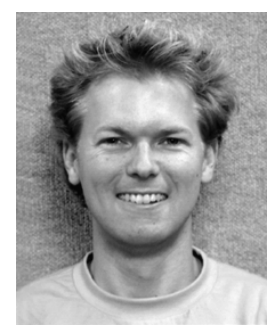

Jamie Evans (S'93-M'98) was born in Newcastle, Australia, in 1970. He received the B.S. degree in physics and the B.Eng. degree in computer engineering from the University of Newcastle, Newcastle, Australia, in 1992 and 1993, respectively, and received the University Medal upon graduation. $\mathrm{He}$ received the M.S. and Ph.D. degrees in electrical engineering from the University of Melbourne, Melbourne, Australia, in 1996 and 1998, respectively, and was awarded the Chancellor's Prize for Excellence in the Ph.D. Dissertation.

From March 1998 to June 1999, he was a Visiting Researcher in the Department of Electrical Engineering and Computer Science at the University of California, Berkeley. In 1999, he became a Lecturer at the University of Sydney, Sydney, Australia, where he stayed until July 2001. Since that time, he has been with the Department of Electrical \& Electronic Engineering at the University of Melbourne, Melbourne, Australia, where he is now an Associate Professor. His research interests are in communications theory, information theory, and statistical signal processing, with current focus on wireless communications networks.

Dr. Evans currently serves on the Editorial Board of the IEEE TRANSACTIONS ON WIRELESS COMMUNICATIONS. 